\title{
Editorial
}

\section{New Developments in Sliding Mode Control and Its Applications 2014}

\author{
Ligang Wu, ${ }^{1}$ Rongni Yang, ${ }^{2}$ Guanghui Sun, ${ }^{1}$ Xudong Zhao, ${ }^{3}$ and Peng Shi ${ }^{4,5}$ \\ ${ }^{1}$ Space Control and Inertial Technology Research Center, Harbin Institute of Technology, Harbin 150001, China \\ ${ }^{2}$ School of Control Science and Engineering, Shandong University, Jinan 250061, China \\ ${ }^{3}$ College of Information and Control Engineering, China University of Petroleum, Qingdao 266555, China \\ ${ }^{4}$ College of Automation, Harbin Engineering University, Harbin, Heilongjiang 150001, China \\ ${ }^{5}$ College of Engineering and Science, Victoria University, Melbourne, VIC 8001, Australia
}

Correspondence should be addressed to Ligang Wu; ligangwu@hit.edu.cn

Received 16 April 2015; Accepted 16 April 2015

Copyright (C) 2015 Ligang Wu et al. This is an open access article distributed under the Creative Commons Attribution License, which permits unrestricted use, distribution, and reproduction in any medium, provided the original work is properly cited.

Sliding mode control (SMC) has been proven to be an effective control strategy for various types of systems since its first appearance in the early 1950s. Due to the excellent robustness, SMC has been successfully applied to a wide variety of real world applications such as robot manipulators, underwater vehicles, spacecrafts, electrical motors, power systems, and automotive engines. Although lots of achievements have been made by using SMC methods and techniques, there are still many systems such as high-order systems, stochastic systems, and switched systems that cannot be easily tackled by using common SMC approaches. With the rapid developments of effective mathematical tools and advanced control technologies, SMC will find more and more applications in practice. At present, it is urgent to solve a number of challenging issues in both theory and application of SMC.

This special issue aims at providing timely discussions on the challenges and trends of SMC of complex dynamic systems. The special issue includes both theory and applicationoriented papers, representing the frontier developments of SMC in recent years. This special issue contains twenty papers most of which are concerned with SMC applications; four papers investigate applications of SMC in practical engineering systems; two articles study tracking problem by using SMC; based on the observer technique, three works are devoted to SMC design for different types of dynamic systems; there are two papers introducing sliding mode controllers to achieve synchronization of chaotic systems and Lorenz systems, respectively; there is also a single article addressing quasi-sliding networked control of systems subject to unbounded disturbance with limited changing rate; another paper proposes design method of active disturbance rejection for variable structure control system; finally, an article designs a mixed variable speed reaching law of sliding mode control for spacecraft tracking system. The contents of this special issue are summarized as follows.

In the application aspect, "Multivariable Super Twisting Based Robust Trajectory Tracking Control for Small Unmanned Helicopter" by X. Fang et al. is devoted to presenting a highly robust trajectory tracking controller for small unmanned helicopter with model uncertainties and external disturbances. "Adaptive Output Tracking Control for Nonlinear Systems with Failed Actuators and Aircraft Flight System Applications" by C. Hou et al. proposes an adaptive failure compensation scheme using output feedback for a class of nonlinear systems with nonlinearities depending on the unmeasured states of systems. "An Improved Optimal Capacity Ratio Design Method for WSB/HPS System Based on Complementary Characteristics of Wind and Solar" by $\mathrm{X}$. Yin et al. proposes an improved capacity ratio design method for stand-alone wind-solar-battery hybrid power system. The proposed method takes full advantages of the complementary characteristics of wind and solar, which can improve power supply reliability while requiring less 
battery capacity. "Performance Analysis of DTC-SVM Sliding Mode Controllers-Based Parameters Estimator of Electric Motor Speed Drive" by F. B. Salem and N. Derbel studies a framework which unifies Direct Torque Control Space Vector Modulation and Variable Structure Control, and the obtained control laws are very sensitive to variations of the stator resistance, the rotor resistance, and the mutual inductance.

Based on sliding mode control method, the following two papers tackle the synchronization problem of two classes of chaotic systems. "Dislocation Synchronization of the Different Complex Value Chaotic Systems Based on Single Adaptive Sliding Mode Controller" by L. Huang et al. studies a new method of the combination of the active control and the single adaptive sliding mode variable structure control to realize the dislocation synchronization of the three-dimensional different complex value chaotic systems based on the active control and the adaptive sliding mode controller. "Synchronization of Lorenz System Based on Fast Stabilization Sliding Mode Control" by X. Guowei et al. applies a sliding mode control approach to Lorenz system based on optimal finite-time convergent and integral sliding mode surface. The robustness of the system can be ensured by the controller which is designed based on integral sliding mode method. An adaptive high-gain K-filter is presented to suppress the nonlinearities while the proposed backstepping adaptive high-gain controller guarantees the stability of the closed-loop system and small tracking errors. Similarly, the SMC is also used to achieve the consensus of a class of multiagent systems in "Second-Order Super-Twisting Sliding Mode Control for Finite-Time Leader-Follower Consensus with Uncertain Nonlinear Multiagent Systems" by N. Liu et al. The paper investigates the consensus tracking problem of the leader-follower multiagent systems via second-order supertwisting sliding mode control approach, and the proposed approach can ensure the finite-time consensus if the directed graph of the nonlinear system has a directed path under the condition that leader's control input is unavailable to any followers.

Furthermore, observer-based sliding mode control attracts some attention in this special issue. "Nonlinear Disturbance-Observer-Based Sliding Mode Control for Flexible Air-Breathing Hypersonic Vehicles" by N. Wang et al. investigates a tracking problem for flexible air-breathing hypersonic vehicles with composite disturbance. A novel composite control strategy is presented for the nonlinear flexible air-breathing hypersonic vehicles model with the composite disturbance, which combines a nonlinear disturbance-observer-based compensator and a dynamicinversion-based sliding mode controller, while "Unknown Disturbance Estimation for a PMSM with a Hybrid Sliding Mode Observer" by G. Chen et al. develops a hybrid sliding mode observer that combines a high-gain feedback and a high-order sliding mode term to identify the timevarying disturbance for a permanent-magnet synchronous motor. "Finite-Time Reentry Attitude Control Using TimeVarying Sliding Mode and Disturbance Observer" by X. Wu et al. presents the finite-time attitude control for reentry vehicle with redundant actuators in consideration of planet uncertainties and external disturbances. "A Nonlinear Exact
Disturbance Observer Inspired by Sliding Mode Techniques" by X. Chen proposes a nonlinear exact disturbance observer by using sliding mode techniques. The proposed method is robust to the type of disturbance and is easy to be implemented.

In addition, some other SMC related topics are also studied in this special issue. "Adaptive Second Order Sliding Mode Control of a Fuel Cell Hybrid System for Electric Vehicle Applications" by J. Liu et al. presents an adaptivegain second-order sliding mode control applied to a hybrid power system for electric vehicle applications. The main advantage of the adaptive second-order sliding mode is that it does not require the upper bound of the uncertainty. "Nonlinear Diversified Processes Monitoring" by Y. Fan et al. is concerned with a new method to classify and establish the monitoring model for diversified processes data with multiscale. "Output Feedback and Single-Phase Sliding Mode Control for Complex Interconnected Systems" by Y.-W. Tsai and V. V. Huynh generalizes a new sliding mode control without reaching phase to solve two important problems in the stability of complex interconnected systems, and the proposed single-phase SMC together with the decentralized output feedback controller can ensure the robust stability of complex interconnected systems. "Quasi-Sliding Networked Control of Systems Subject to Unbounded Disturbance with Limited Rate of Change" by A. Bartoszewicz and P. Latosiński is concerned with network based sliding mode control of linear plants with state measurement delay. In order to combat the unpredictable disturbance in the environment with state measurement delay, a novel sliding mode controller is introduced. "A New SVM Multiclass Incremental Learning Algorithm" by Y. Qin et al. investigates a new support vector machine multiclass incremental learning algorithm, and "Zero-Disturbance Control of Free-Floating Space Manipulators Using Integral-Type Sliding Mode Control” by H. Li and R. Li describes a zero-disturbance control method for free-floating space manipulators operating in task space. An explicit direct relationship between the spacecraft attitude quaternions and the manipulator joint variables is established using nonholonomic constraints of the angular momentum conservation. "A Mixed Variable Speed Reaching Law of Sliding Mode Control for Spacecraft Tracking System" by Y. Zhang and Y.-X. Zhao is concerned with a mixed variable speed reaching law of SMC and a controller for spacecraft tracking system, and a mixed variable speed reaching law of SMC and a controller for spacecraft tracking system was investigated. "Statistical Analysis of Nonlinear Processes Based on Penalty Factor" by Y. Zhang et al. presents a new process monitoring approach for handling the nonlinear monitoring problem in the electrofused magnesia furnace. "Design Method of Active Disturbance Rejection Variable Structure Control System" by Y. Wu et al. provides lines cluster approaching mode control method to improve the parameter simplicity and structure optimization of the control system.

In summary, some basic but challenging problems related to SMC have been solved in this special issue, which also reflect some future study directions of SMC. Certainly, the selected topics and papers are not a comprehensive 
representation of the area of this special issue. However, they represent multifaceted knowledge and we hope the readers will find them useful as much as we do.

\section{Acknowledgments}

We would like to express our great appreciation to the authors for their remarkable contributions in assisting us. Finally, all reviewers' fundamental works and time on these papers are also very greatly acknowledged.

Ligang Wu Rongni Yang Guanghui Sun Xudong Zhao Peng Shi 


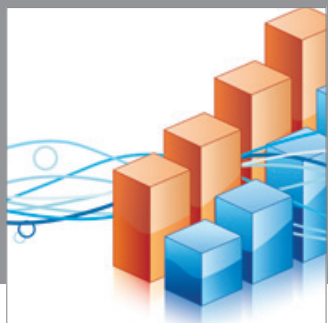

Advances in

Operations Research

mansans

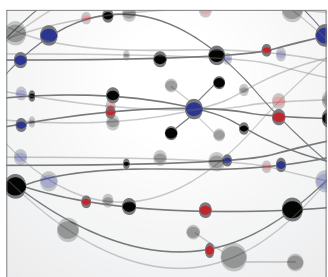

The Scientific World Journal
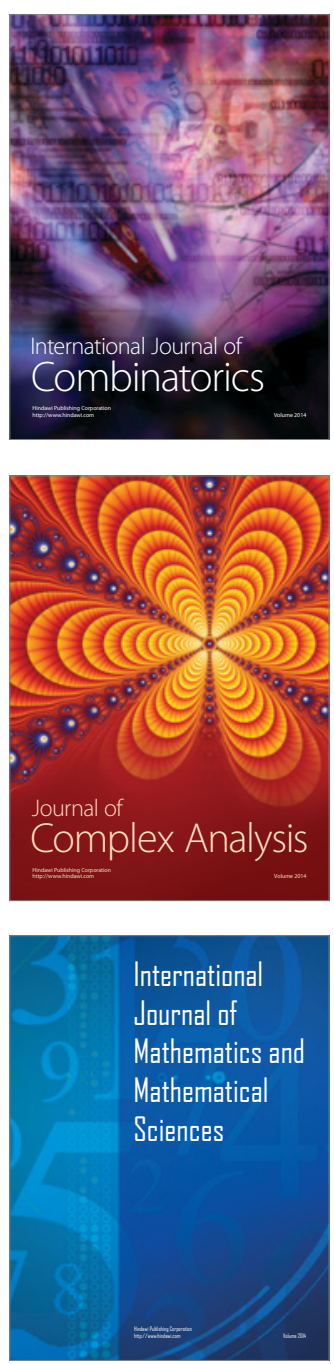
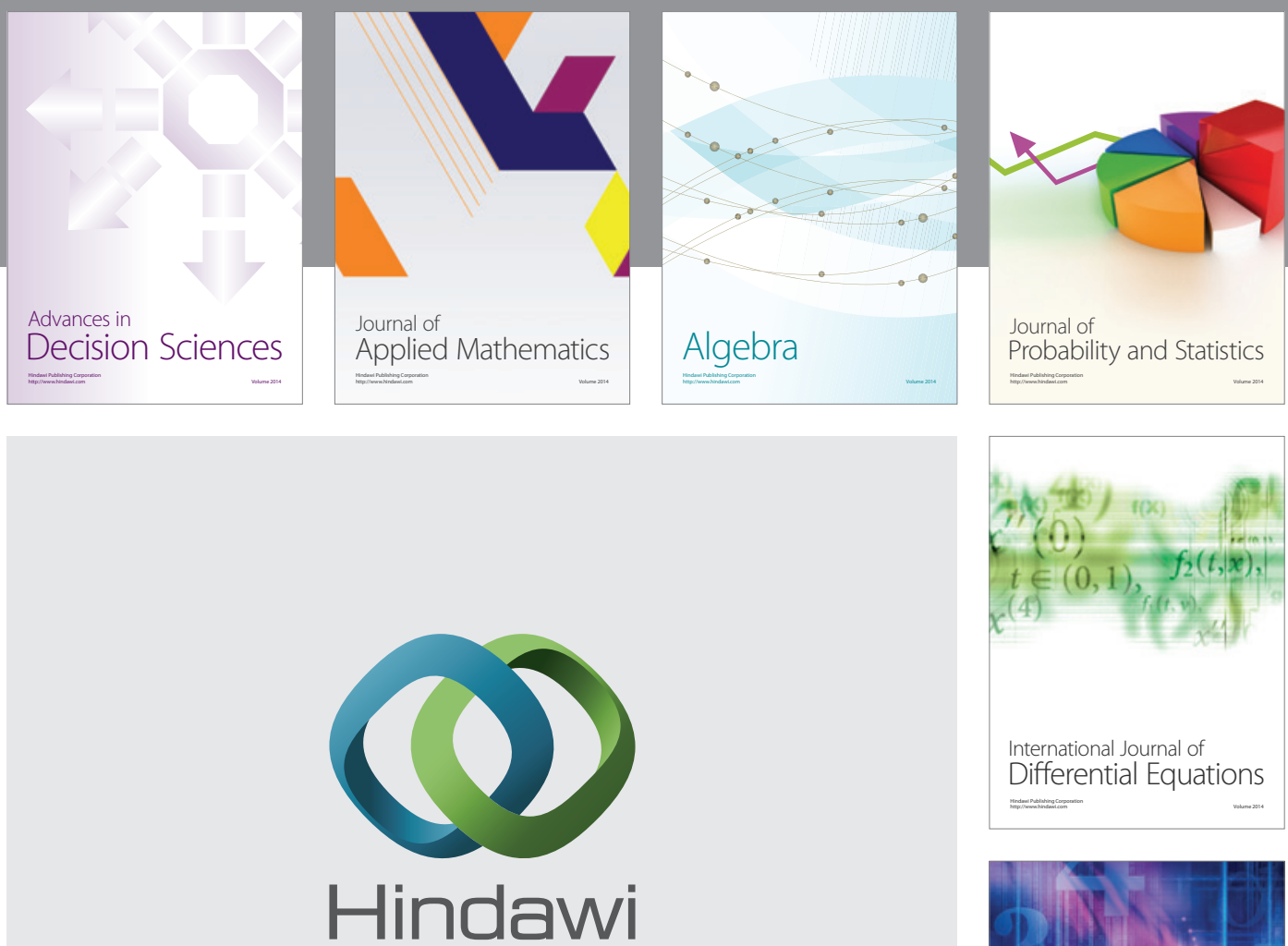

Submit your manuscripts at http://www.hindawi.com
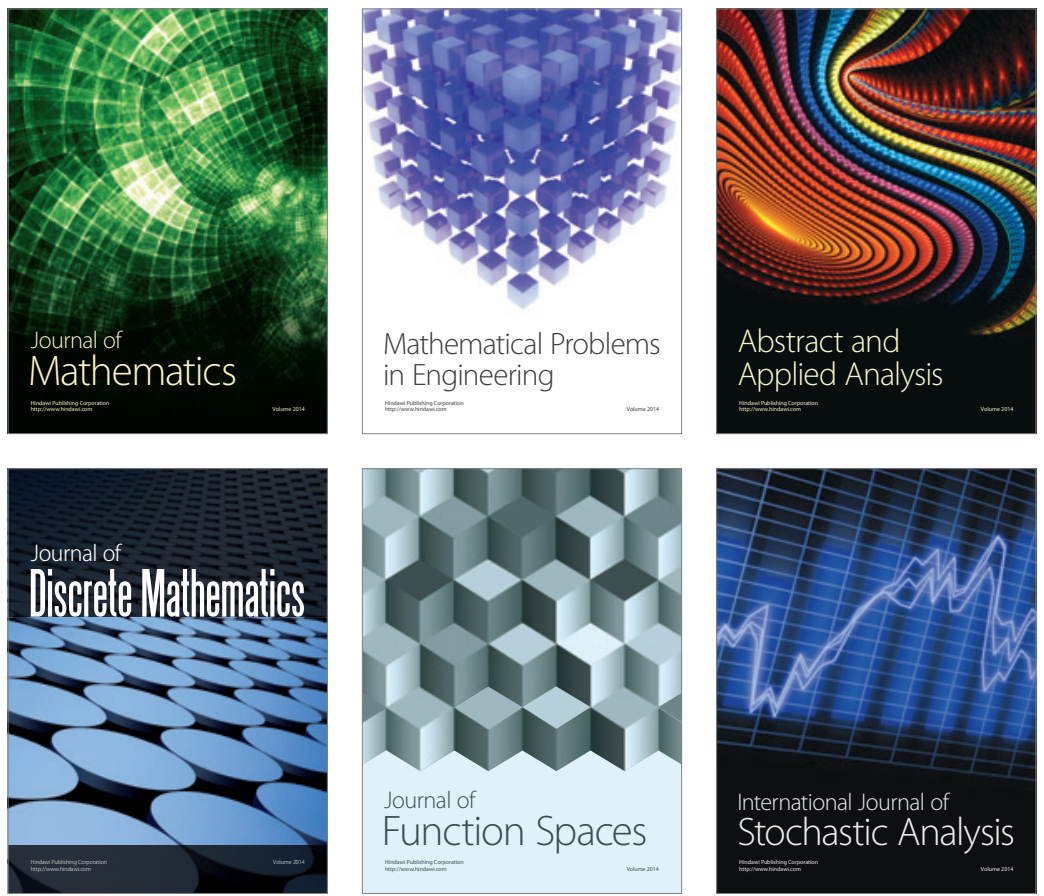

Journal of

Function Spaces

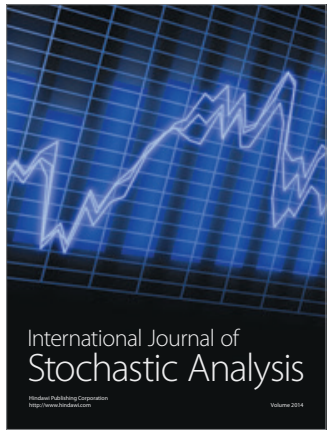

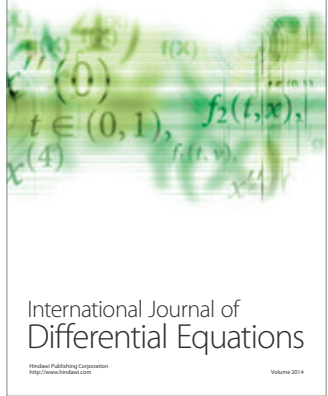
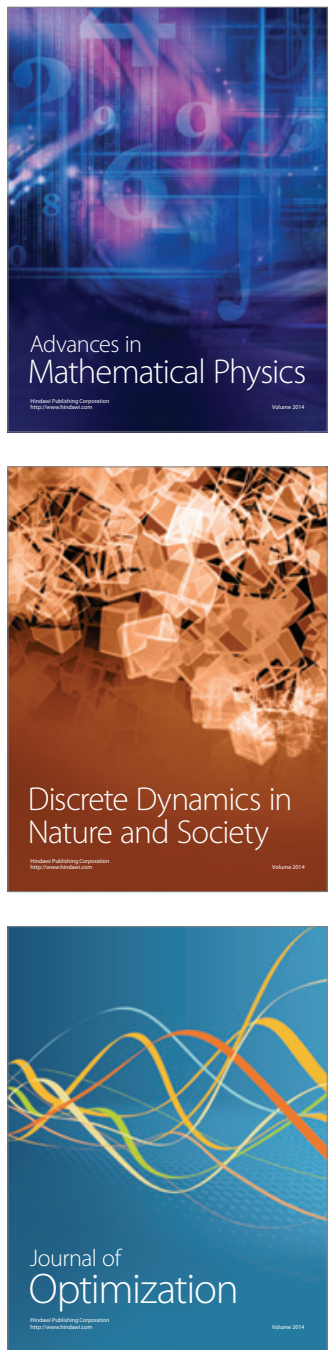